\title{
Inverse Method based on Cosine Integral Transforms to Estimate 2-D Heat Flux and Thermal Diffusivity
}

\author{
by A. AOUALI*, S. CHEVALIER*, A. SOMMIER*, J.C. BATSALE* and C. PRADERE*
}

* I2M TREFLE, UMR CNRS-UB-ENSAM 5295, Esplanade des Arts et Métiers, 33405 Talence Cedex, France

\section{Abstract}

The estimation of physical properties in complex materials and boundary conditions such as flux or temperature at the interface require the development of inverse method. When used in combination of imaging techniques using an IR camera, such methods can be used to estimate the transient spatial distribution of heat flux, temperature and material physical properties [1]. The choice of the inverse method directly linked to the parameters that need to be identified. In this work, we report an inverse method based on integral cosine transforms to estimate the spatial distribution and amplitude of heat flux, as well as the in-plane diffusivities in a homogenous copper block.

An imaging experiment was performed were the temperature field at the surface of the copper slab was measured using an IR camera (see Figure 1). To estimate the heat flux at rear face of the copper slab, an inverse method based on an analytical asymptotic solution of heat equation in the Fourier space is developed [2]. By using two cosine integral transforms of the temperature measurement at the surface of a material with known dimensions $\left(L_{x} \times L_{y} \times e\right)$, and known properties, the density $(\rho)$, as well as the calorific capacity $(C p)$ of the material. The advantages of the inverse methods into the Fourier space are solution to the equation is a product of the initial condition and the exponential of the product of the spatial frequencies, the longitudinal diffusivities and the time, on the other hand, because we obtain a spatial frequency distribution (possibility of spatial filtering). Finally, by applying the logarithm function to the solution obtained, we will have a first order polynomial, with the initial condition as intercept, and the longitudinal diffusivities as slope.

The proposed inverse method has been validated numerically where the spatial distribution of the heat flux was estimated. The next step is to use the method with a temperature field measured by an IR camera. In this communication, the full presentation of the inverse method and the experimental setup will be presented. A discussion about the limitation of the heat flux and diffusivity estimation will be done in particularly concerning the noise in the experimental data.

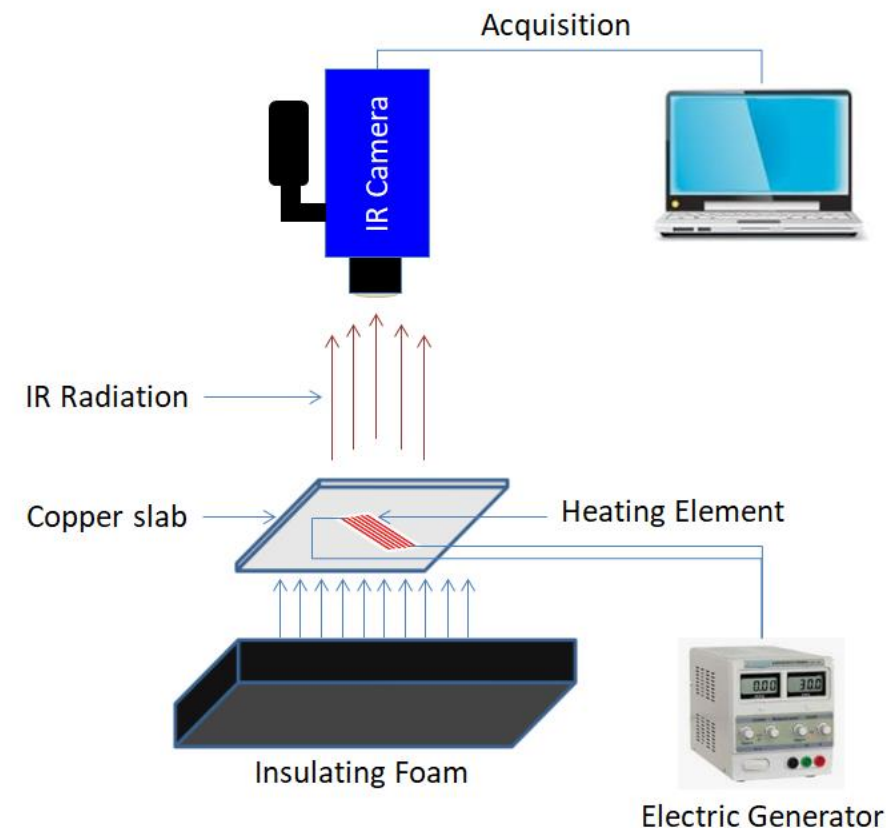

Fig. 1. Set-up used for the heat flux and diffusivity estimation 


\section{REFERENCES}

[1] J.-C. Krapez, L. Spagnolo, M. Frieß, H.-P. Maier et G.U. Neuer : Measurement of in-plane diffusivity in nonhomogeneous slabs by applying flash thermography. International Journal of Thermal Sciences, 43(10):967977, 2004

[2] J.C. BATSALE, D. MAILLET et A. DEGIOVANNI., "Extension de la méthode des quadripôles thermique à l'aide de transformations intégrales, calcul du transfert thermique au travers d'un défaut plan bidimensionnel". Heat Mass Transfer, vol. 37, 1994. 